\title{
A new half-sweep algebraic multigrid (HSAMG) algorithm for two-point boundary problems
}

\begin{abstract}
The primary goal of this paper is to propose a new half-sweep algebraic multigrid (HSAMG) algorithm using the finite difference approximation equation for solving two-point boundary problems. The basic idea and formulation of the HSAMG algorithm are inspired by the concept of the half-sweep multigrid method. Some computational experiments have been conducted in order to show that the HSAMG method is superior to the standard algebraic method.
\end{abstract}

Keyword: Half-sweep algebraic multigrid (HSAMG); Two-point boundary problems 